\title{
The Magnetic Charge Phenomenon: Comparison of Theory and Experiment.
}

\author{
A. M. R. MAGNoN \\ Département de Mathématiques, Université Blaise Pascal \\ Clermont II, BP 45, 63170 Aubière, France \\ (Nuovo Cimento A, 101, 671 (1989)) \\ PACS 12.20.Hx - Unified field theories and models.
}

On p. 671 , third line of the summary, "the" should be "that".

On p. 673 , fifteenth line from the bottom, $S^{2}(x R)$ should be $S^{2}(\times R)$.

On p. 674, sixth line, $\chi=x+2 \Omega^{-1} \Omega$ should be replaced by $\chi=x+2 \Omega^{-1} \mathscr{P}_{\xi} \Omega$.

On p. 674, bottom line and on p. 675 , twelfth line from the top, "provided" should be "provide".

On p. 675 , fourth line, «empting" should be «tempting».

On p. 675,18 th line $\nabla c_{t} d$ should be $\nabla^{c} t^{d}$.

(C) by Società Italiana di Fisica

Proprietà letteraria riservata

Direttore responsabile: RENATO ANGELO RICCI

Questo fascicolo è stato realizzato in fotocomposizione dalla Monograf, Bologna e stampato dalla tipografia Compositori, Bologna nel mese di agosto 1989 